\title{
New Thiazolidinones, Thiazolines and Thiopyrimidines from 3,5-Diphenylcyclohex-2-enone
}

\author{
M. A. Metwally ${ }^{*}$ E. Abdel-Galil, F. A. Amer, A. M. Abdallah \\ Chemistry Department, Faculty of Science, Mansoura University, Mansoura, 35516, Egypt
}

\begin{abstract}
Diphenylcyclohex-2-enylidene)hydrazine 3 was prepared and used as a key intermediate for the synthesis of thiazolidin-5-one 6, thiazolidn-4-one 7, thiazolines 8a,b, 9 and thioxodihydropyrimidine 10. Base catalyzed Knoevenagel condensation of compounds 6,7 and 10 with different aldehydes gave the arylidene derivatives $11 \mathrm{a}, \mathrm{b}, 12 \mathrm{a}, \mathrm{b}$ and 13a,b respectively. Treatment of compounds 6, 7 and 10 with different aromatic diazonium salts gave azodye derivatives 14a,b, 15a,b and 16a,b. The newly synthesized compounds were characterized by IR, ${ }^{1} \mathrm{H}-\mathrm{NMR}$ and mass spectral data.
\end{abstract}

Keywords Thiazoloidinones, Thiazolines and Thiopyrimidines

\section{Introduction}

In recent years, thiazolidinones and their derivatives have become among the most extensively investigated compounds. They constitute an important five-membered heterocycles, having valuable biological activities in the areas of medicine and agriculture[1]. They have found uses, for example, as bactericidal[2,3], fungicidal[4,5], insecticidal [6,7], anticonvulsant[8-11], tuberculostatic[12,13], herbicidal $[14,15]$, antiviral[16,17], antiprotozoal[18], antimalarial[19], antimicrobial[20,21], anti- inflammatory[22,23], antitumor[24], antitubercular agents[25] and specially as anti-HIV agents[26]. The diversity of biological and physiological activities of organic sulfur heterocycles may be attributed to the presence of the NCS fragment, which is characteristicof thiazoles, thiazolines, and thiazolidines[27]. In view of the above findings and as an extension of our studies[28-34] aiming to the synthesis of different thiazoles, thiazolodinones and thiazolines, of expected pharmaceutical interest, we report here the reactivity of potassium $N^{\prime}-3,5-$ diphenylcyclohex-2-enylidene- $N$-phenylcarbamoyl hydrazonothionate 4 toward different $\alpha$-halogenated compounds.

\section{Results and Discussion}

The key intermediate, 3 required for the synthesis of the title compounds was prepared according to the procedure outlined in the Scheme 1. For the synthesis of 3, reaction sequence including the first step decarboxylation of ethyl 2-oxo-4,6-diphenylcyclohex-3-ene carboxylate 1 either by a

* Corresponding author:

mamegs@mans.edu.eg (M. A. Metwally)

Published online at http://journal.sapub.org/ajoc

Copyright (C) 2012 Scientific \& Academic Publishing. All Rights Reserved previously reported procedure [35] with $\mathrm{NaOH}$ solution or our new methodology using acetic acid gave3,5- diphenylcyclohex-2-enone 2 . This was reacted with hydrazine hydrate to afford 1-(3,5-diphenylcyclohex-2-enylidene) hydrazine 3 in excellent yield. The structures of the synthesized compounds ( 2 and 3 ) were confirmed by IR, ${ }^{1} \mathrm{H}-\mathrm{NMR}$ and mass spectral analyses. The IR spectrum of compound 2 no absorption was appeared for the ester group. The mass spectrum of 2 showed the molecular ion peak at $\mathrm{m} / \mathrm{z}=248$ $\left(\mathrm{M}^{+}, 100 \%\right)$ which is equivalent to the molecular formula $\left(\mathrm{C}_{18} \mathrm{H}_{16} \mathrm{O}\right)$. The mass spectrum of compound 3 showed the molecular ion peak at $\mathrm{m} / \mathrm{z}=262\left(\mathrm{M}^{+}, 100 \%\right)$, corresponding to the molecular formula $\left(\mathrm{C}_{18} \mathrm{H}_{18} \mathrm{~N}_{2}\right)$.

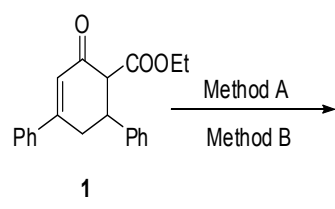

Method $\mathrm{A}: \mathrm{NaOH}, \mathrm{CH}_{3} \mathrm{OH}$, reflux $3 \mathrm{~h}$

Method $\mathrm{B}: \mathrm{AcOH}$, reflux $6 \mathrm{~h}$

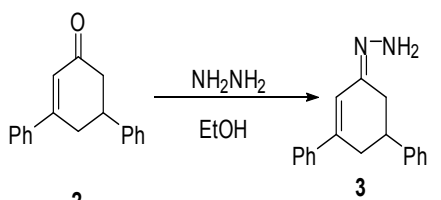

3

Scheme 1
The base promoted nucleophilic addition of 1-(3,5- diphenylcyclohex-2-enylidene)hydrazine 3 to an equimolar amount of phenyl isothiocyanate in dry DMF containing potassium hydroxide afforded the corresponding non isolable potassium $N^{\prime}$-3,5-diphenylcyclohex-2-enylidene- $N$ phenylcarbamoylhydrazonothionate 4 . In situ cyclization of intermediate 4 with chloroacetyl chloride afforded the corresponding 2-(3,5-diphenylcyclohex-2-enylidene)hydrazono) 3 -phenylthiazolidin-5-one 6 . The cyclized product 6 was also obtained upon treatment of thiosemicarbazone 5 with chloroacetyl chloride in dry DMF containing potassium hydroxide. The IR spectrum of compound 6 showed cyclic carbonyl absorption at $1680 \mathrm{~cm}^{-1}$. The ${ }^{1} \mathrm{H}-\mathrm{NMR}$ spectrum of 
6 showed a singlet signal equivalent to two protons at $\delta 4.10$ due to the methylene protons at $\mathrm{C}-4$ of the thiazolidine ring $\left(\mathrm{CO}-\mathrm{CH}_{2}-\mathrm{N}\right)$.

New heterocyclic ring systems namely 2-(3,5- diphenylcyclohex-2-enylidene) hydrazono) - 3 - phenylthiazolidin- 4one 7, 2-(3,5-diphenylcyclohex-2-enylidene)hydrazono)-4substituted-3-phenyl-2,3-dihydrothiazole 8a,b,2-(3,5-diphenylcyclohex-2-enylidene)hydrazono)-3-phenylthiazolidin-4imine 9 were prepared by in situ cyclization of such intermediate 4 with chloroacetic acid or ethyl bromoacetate, $\alpha$-halogenated ketones namely (chloroacetone, phenacyl bromide) and chloroacetonitrile respectively. On the other hand, the thiosemicarbazone 5 was achieved by treating intermediate 4 with dil.HCl. The IR spectrum of compound 5 exhibits two bands at $3370,3413 \mathrm{~cm}^{-1}$ due to $\mathrm{NH}$ groups. (c.f. experimental). Thiosemicarbazone 5 was treated with malonic acid in the presence of acetyl chloride to give the corresponding 2-thioxopyrimidine-4,6-dione derivative 10 .

Structures 7-10 were established on the basis of both spectral data and elemental analyses. For example, The IR spectrum of 7 showed absorption band at $1727 \mathrm{~cm}^{-1}$ attributed to $\mathrm{C}=\mathrm{O}$ groups. Its ${ }^{1} \mathrm{H}-\mathrm{NMR}$ spectrum displayed a singlet signal equivalent to two protons at $\delta 3.97\left(\mathrm{COCH}_{2}-\mathrm{S}\right)$. The mass spectrum measurement gave an evidence for the proposed structure, which showed the molecular ion peak at $\mathrm{m} / \mathrm{z}=437\left(\mathrm{M}^{+}, 100 \%\right)$, corresponding to the molecular formula $\left(\mathrm{C}_{27} \mathrm{H}_{23} \mathrm{~N}_{3} \mathrm{OS}\right)$. (c.f. experimental). The ${ }^{1} \mathrm{H}-\mathrm{NMR}$ spectrum of 9 revealed singlet signal due to methylene group $\left(-\mathrm{S}-\mathrm{CH}_{2}-\mathrm{C}=\mathrm{NH}\right)$ at $3.96 \mathrm{ppm}$.

The behaviour of compounds 6,7 and 10 toward aromatic aldehydes was investigated Scheme 3. It was found that, 2-((3,5-Diphenylcyclohex-2-enylidene)hydrazono)-3-pheny lthiazolidin-5-one 6 reacted with benzaldehyde in ethanol in the presence of piperidine to afford the corresponding 4benzylidiene-2-((3,5- diphenylcyclohex-2- enylidene) hydr- azono)-3-phenylthiazolidin-5-one11a. Similarly, 2-((3,5-diphenylcyclohex-2-enylidene) hydrazono)-3-phenylthiazolidin- 4- one 7 and 1-(3,5- diphenylcyclohex- 2- enylidene amino)-3-phenyl-2-thioxodihydropyrimdine-4,6- $(1 H, 5 H)$-di one 10 when reacted with aromatic aldehydes under the same experimental conditions yielded the corresponding 5arylidine-2-((3,5-diphenylcyclohex-2-enylidene)hydrazono) -3-phenylthiazolidin-4-one 12a,b and 5-arylidine-1- (3,5diphenylcyclohex-2-enylideneamino)- 3- phenyl- 2- thioxodihydropyrimdidne-4,6-(1H,5H)-dione 13a,b respectively.

Structures $11 \mathrm{a}, \mathrm{b}-13 \mathrm{a}, \mathrm{b}$ were confirmed on the basis of analytical and spectral data. The ${ }^{1} \mathrm{H}-\mathrm{NMR}$ spectra of compounds $(11 a, b-13 a, b)$ were characterized by the absence of the singlet signal of methylene $\left(-\mathrm{CH}_{2}-\right)$ group of thiazolidinones 6, 7 and thioxo dihydropyrimdidne-4,6- $(1 H, 5 H)$-dione 10. The Knoevenagel condensation of 2-((3,5 - diphenylcyclohex-2-enylidene ) hydrazono)-3-phenylthiazolidin-4-one 7 with aromatic aldehydes provide only the $Z$ isomer as determined by the chemical shift of the methine proton in $12 \mathrm{a}$ at $\delta=7.78 \mathrm{ppm}$ as singlet signal. The exclusive formation of thermodynamically stable $\mathrm{Z}$ isomer is in agreement with which was reported similar structures in the literature [36,37].

As a continuation of our studies on the synthesis of heterocyclic azo dyes[33,34,38,39], scheme 3 the behaviour of compounds 6,7 and 10 toward arenediazonium chlorides was studied. Thus, 6,7 and 10 was reacted with $p$ - chlorobenzendiazonium chloride and $p$-methoxybenzendiazonium chloride in pyridine to give the corresponding azo - derivatives $14 a, b, 15 a, b$ and $16 a, b$ respectively. Confirmation of the structures $14 \mathrm{a}, \mathrm{b}-16 \mathrm{a}, \mathrm{b}$ was based on analytical and spectral data. ${ }^{1} \mathrm{H}-\mathrm{NMR}$ of $14 \mathrm{a}, \mathrm{b}$ were characterized by the presence of the singlet signal of methyne $\left(\mathrm{C}_{4}-\mathrm{H}\right)$ group at $\delta=$ $3.5 \mathrm{ppm}$.

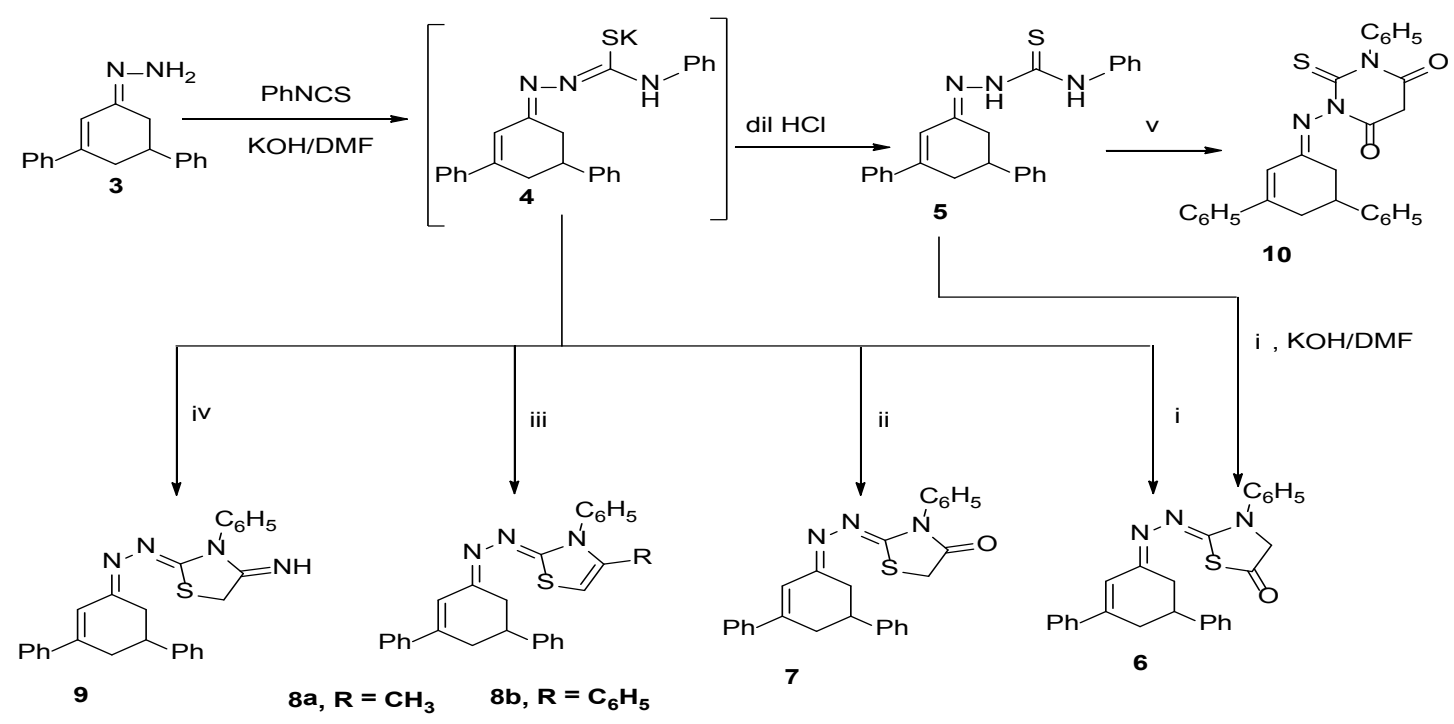

\footnotetext{
Reagents : i - chloroacetylchloride ii- chloroacetic acid or ethyl bromoacetate iii - a) chloroacetone, b) phenacyl bromide v- malonic acid and acetyl chloride

iv- chloroacetonitrile
} 


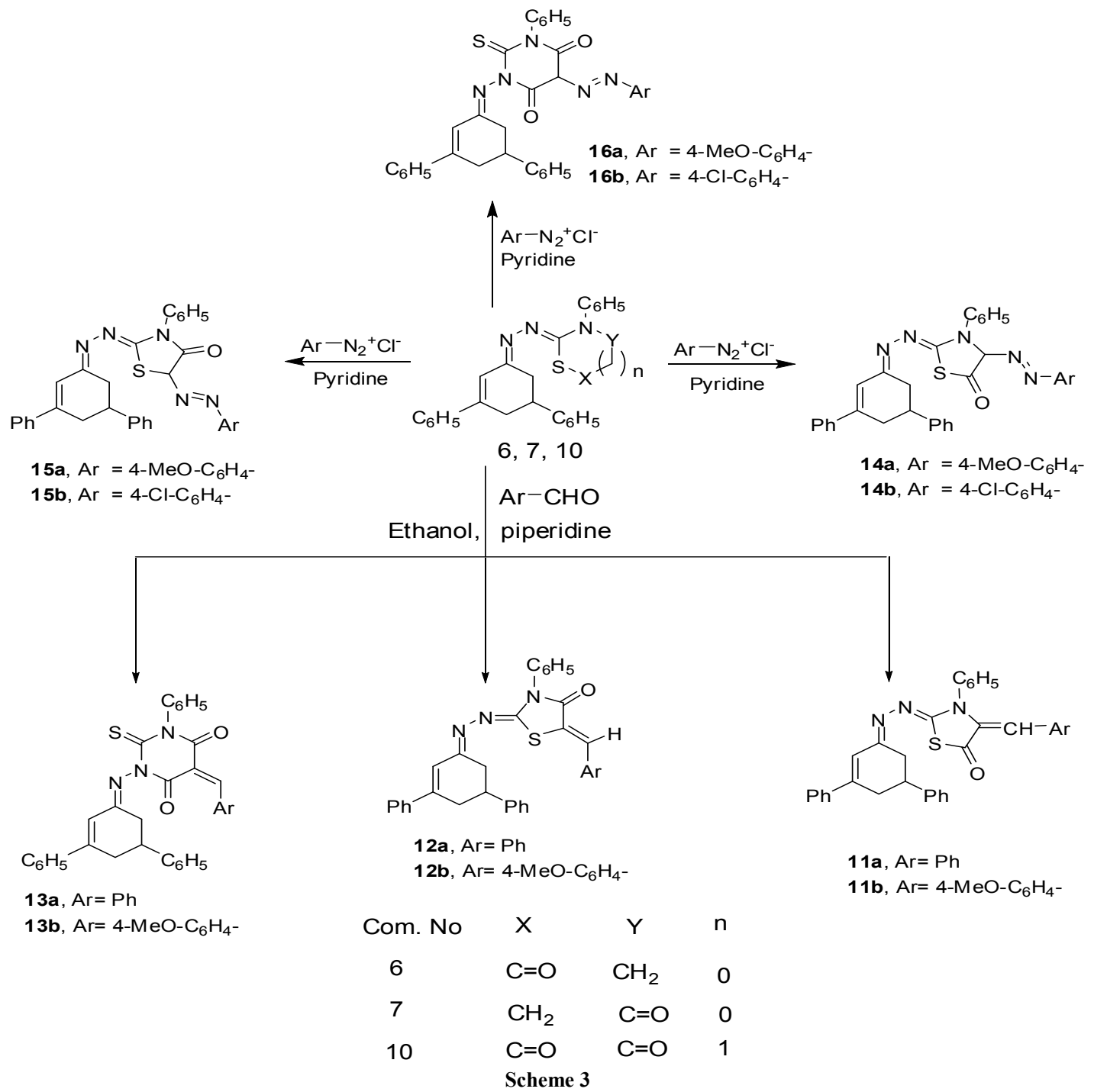

\section{Conclusions}

In conclusion, conducting reactions using thiocarbamoyl derivatives is an effective method for reactions leading to various $\mathrm{C}-\mathrm{C}$ and $\mathrm{C}-\mathrm{N}$ bond forming reactions. New thiazolidin-5-ones and 4-ones are of great importance, which were synthesized. The corresponding arylidines and arylazo derivates were also prepared.

\section{Experimental}

All melting points were determined on Gallenkamp electric melting point apparatus and were uncorrected. Elemental analyses were carried out at the Microanalytical Unit at Faculty of Science, Mansoura University, Egypt. IR spectra were recorded on a Mattson 5000 Fourier transform infrared (FTIR) spectrometer. The ${ }^{1} \mathrm{H}$ NMR spectra were measured on a Bruker WP 300 in $\mathrm{CDCl}_{3}$ and DMSO- $d_{6}$ as solvent using tetramethylsilane (TMS) as an internal standard. Mass spectra were recorded on a Finnigan MAT 212 instrument at the Microanalytical Unit at Faculty of Science, Cairo Uni- versity, Egypt. Compound 1 and 2 were prepared according to a previously reported method [35].

\section{3,5-Diphenylcyclohex-2-en-1-one 2. (Method B)}

Refluxing of $1(3.2 \mathrm{~g}, 10 \mathrm{mmol})$ in $(20 \mathrm{ml})$ glacial acetic acid for $6 \mathrm{~h}$. The reaction mixture was poured onto crushed ice then the precipitate was filtered off, dried, and recrystallized from ethanol to give buff powder, $\mathrm{mp} 82^{\circ} \mathrm{C}[$ lit. $35 \mathrm{mp}$ 81-83. Method A], yield 95\%, IR (KBr) (vmax, $\left.\mathrm{cm}^{-1}\right)$ : 1658 $(\mathrm{C}=\mathrm{O}) . \mathrm{m} / \mathrm{z} 248\left(\mathrm{M}^{+}, 100 \%\right)$.

\section{(3,5-Diphenylcyclohex-2-enylidene)hydrazine 3}

A mixture of $2(2.48 \mathrm{~g}, 10 \mathrm{mmol})$ and hydrazine hydrate $(0.6 \mathrm{~mL}, 12 \mathrm{mmol})$ in absolute ethanol $(15 \mathrm{~mL})$ was refluxed for $3 \mathrm{~h}$. The reaction mixture was left to cool. The precipitated solid was filtered off, dried and recrystallized from ethanol to afford hydrazone derivative 3. Yellow powder, $\mathrm{mp}$ $156^{\circ} \mathrm{C}$, yield $85 \%$, IR $(\mathrm{KBr})\left(\operatorname{vmax}, \mathrm{cm}^{-1}\right): 3353-3316\left(\mathrm{NH}_{2}\right)$, $1614(\mathrm{C}=\mathrm{N}) .{ }^{1} \mathrm{H}-\mathrm{NMR}\left(\mathrm{CDCl}_{3}\right) \delta \mathrm{ppm}=2.21-2.38(\mathrm{~m}, 2 \mathrm{H}$, $\left.\mathrm{C}_{4}-\mathrm{H}\right), 2.65-2.73\left(\mathrm{~m}, 2 \mathrm{H}, \mathrm{C}_{6}-\mathrm{H}\right), 2.93-3.07\left(\mathrm{~m}, 1 \mathrm{H}, \mathrm{C}_{5}-\mathrm{H}\right)$, $6.59\left(\mathrm{~d}, 1 \mathrm{H}, J=3 \mathrm{~Hz}, \mathrm{C}_{2}-\mathrm{H}\right), 7.12-7.57(\mathrm{~m}, 10 \mathrm{H}, \mathrm{Ar}-\mathrm{H}), 9.46$ (s, 2H, NH $\mathrm{NH}_{2}$. MS: (m/z, \%): 262 (100), 245 (54.6), 202 (23.7), 143 (34.2), 115 (36.4), 91 (75.6), 77 (48.05). Anal. Calcd. For $\mathrm{C}_{18} \mathrm{H}_{18} \mathrm{~N}_{2}$ (262.35): C, 82.41, H, 6.92, N, 10.68\%. 
Found: C, 82.53, H, 6.97, N, 10.74\%.

2-(3,5-Diphenylcyclohex-2-enylidene)- $N$-phenylhydrazin ecarbothioamide 5 .

To a vigorously stirred solution of $3(2.62 \mathrm{~g}, 10 \mathrm{mmol})$ in dry DMF $(10 \mathrm{~mL})$ at room temperature, potassium hydroxide $(0.56 \mathrm{~g}, 10 \mathrm{mmol})$ and phenylisothiocyanate $(1.2 \mathrm{~mL}, 10 \mathrm{~mol})$ were added simultaneously over $30 \mathrm{~min}$. Stirring was continued for a further $30 \mathrm{~min}$. The reaction mixture was poured into ice cold water and acidified with dilute $\mathrm{HCl}$. The solid obtained was filtered off, dried and recrystallised from ethanol to afford 5 . Pale yellow powder, $\mathrm{mp} 223^{\circ} \mathrm{C}$, yield $88 \%$, IR (KBr) $\left(\operatorname{vmax}, \mathrm{cm}^{-1}\right): 3192(2 \mathrm{NH}), 1614(\mathrm{C}=\mathrm{N})$. ${ }^{1} \mathrm{H}-\mathrm{NMR} \delta \mathrm{ppm}=2.31-2.37\left(\mathrm{~m}, 2 \mathrm{H}, \mathrm{C}_{4}-\mathrm{H}\right), 2.65-2.73(\mathrm{~m}, 2 \mathrm{H}$, $\left.\mathrm{C}_{6}-\mathrm{H}\right), 2.83-2.87(\mathrm{~m}, 1 \mathrm{H}, \mathrm{C} 5-\mathrm{H}), 6.59\left(\mathrm{~d}, 1 \mathrm{H}, \mathrm{J}=3 \mathrm{~Hz}, \mathrm{C}_{2}-\mathrm{H}\right)$, 6.99-7.54 (m, 15H, Ar-H), 8.53, $5.13(\mathrm{~s}, 2 \mathrm{H}, 2 \mathrm{NH})$. MS: (m/z, \%): 397 (1.60), 396 (3.70), 395 (9.91), 379 (7.1), 364 (351), 304 (8.1), 262 (100), 245 (70.2), 232 (26.5), 202 (16.5), 143 (34.2), 128 (36.2), 115 (38.7), 91 (75.6), 77 (27.05). Anal. Calcd. for $\mathrm{C}_{25} \mathrm{H}_{23} \mathrm{~N}_{3} \mathrm{~S}$ (397.54): C, 75.53, H, 5.83 , N, 10.57\%. Found: C, 75.61, H, 5.88, N, 10.61\%.

2-(3,5-Diphenylcyclohex-2-enylidene)hydrazono)-3-phen ylthiazolidin-5-one 6

A mixture of compound $5(3.97 \mathrm{~g}, 10 \mathrm{mmol})$, chloroacetyl chloride $(0.79 \mathrm{ml}, 10 \mathrm{mmol})$ and potassium hydroxide $(0.56$ $\mathrm{g}, 10 \mathrm{mmol})$ in dry DMF $(10 \mathrm{~mL})$ was vigorously stirred at room temperature for $6 \mathrm{~h}$. The reaction mixture was poured into ice cold water. The precipitated solid was filtered off, dried and crystallized from ethanol to afford 6. Yellow powder, $\mathrm{mp} 121^{\circ} \mathrm{C}$, yield $85 \%$, IR $(\mathrm{KBr})\left(\operatorname{vmax}, \mathrm{cm}^{-1}\right): 1680$ $(\mathrm{C}=\mathrm{O}), 1612(\mathrm{C}=\mathrm{N}) .{ }^{1} \mathrm{H}-\mathrm{NMR} \quad \delta p p m=2.78-2.89(\mathrm{~m}, 2 \mathrm{H}$, $\left.\mathrm{C}_{4}-\mathrm{H}\right), 2.97-3.2\left(\mathrm{~m}, 2 \mathrm{H}, \mathrm{C}_{6}-\mathrm{H}\right), 3.46-3.62\left(\mathrm{~m}, 1 \mathrm{H}, \mathrm{C}_{5}-\mathrm{H}\right), 4.1$ (s, $2 \mathrm{H}, \mathrm{CH}_{2}$ thiazole), $6.58\left(\mathrm{~d}, 1 \mathrm{H}, J=3 \mathrm{~Hz}, \mathrm{C}_{2}-\mathrm{H}\right), 6.96-7.57$ (m, 15H, Ar-H). MS: (m/z, \%): 437 (2.25), 408 (2.1), 381 (2.0), 262 (9.5), 212 (7.5), 141 (6.1), 105 (7.2), 93 (100), 77 (16.0). Anal. Calcd. for $\mathrm{C}_{27} \mathrm{H}_{23} \mathrm{~N}_{3} \mathrm{OS}$ (437.56): C, 74.11, H, 5.30 , N, 9.60\%. Found: C, 74.16, H, 5.38, N, 9.66\%.

\section{Thiazolidine derivatives $6,7,8 \mathrm{a}, \mathrm{b}$ and 9}

To a vigorously stirred solution of hydrazone $3(2.62 \mathrm{~g}, 10$ $\mathrm{mmol})$ in dry DMF $(10 \mathrm{~mL})$ at room temperature, phenylisothiocyanate $(1.2 \mathrm{~mL}, 10 \mathrm{mmol})$ and potassium hydroxide $(0.56 \mathrm{~g}, 10 \mathrm{mmol})$ were added simultaneously over $30 \mathrm{~min}$. Stirring was continued for a further $30 \mathrm{~min}$. $\alpha$-Halo compounds namely, chloroacetyl chloride $(0.79 \mathrm{ml}, 10 \mathrm{mmol})$, chloroacetic acid $(0.94 \mathrm{~g}, 10 \mathrm{mmol})$ or ethyl bromoacetate $(1.1 \mathrm{ml}, 10 \mathrm{mmol})$, chloroacetone $(0.79 \mathrm{ml}, 10 \mathrm{mmol})$, phenacyl bromide $(2.0 \mathrm{~g}, 10 \mathrm{mmol})$ and chloroacetonitrile $(0.63 \mathrm{ml}, 10 \mathrm{mmol})$ were added drop wise to the reaction mixture with stirring at $5-10^{\circ} \mathrm{C}$ for $3 \mathrm{~h}$ then, poured into ice-water, the solid obtained was filtered off, dried and recrystallised from ethanol to afford $6,7,8 \mathrm{a}, \mathrm{b}$ and 9 , respectively.

\section{2-(3,5-Diphenylcyclohex-2-enylidene)hydrazono)-3-phen} ylthiazolidin-4-one 7

Buff powder, m.p. $196^{\circ} \mathrm{C}$, yield $70 \%$, IR ( $\left.\mathrm{KBr}\right)$ (vmax, $\left.\mathrm{cm}^{-1}\right): 1727(\mathrm{C}=\mathrm{O}), 1612(\mathrm{C}=\mathrm{N}) .{ }^{1} \mathrm{H}-\mathrm{NMR} \delta \mathrm{ppm}=2.79-2.90$ $\left(\mathrm{m}, 2 \mathrm{H}, \mathrm{C}_{4}-\mathrm{H}\right), 2.98-3.07\left(\mathrm{~m}, 2 \mathrm{H}, \mathrm{C}_{6}-\mathrm{H}\right), 3.17-3.52(\mathrm{~m}, 1 \mathrm{H}$, $\left.\mathrm{C}_{5}-\mathrm{H}\right), 3.99\left(\mathrm{~s}, 2 \mathrm{H}, \mathrm{CH}_{2}\right.$ thiazole) $6.51\left(\mathrm{~d}, 1 \mathrm{H}, J=3 \mathrm{~Hz}, \mathrm{C}_{2}-\mathrm{H}\right)$,
7.09-7.56 (m, 15H, Ar-H). MS: (m/z, \%): 439 (15.0), 438 (28.4), 437 (100), 381 (15.0), 345 (21.2), 288 (26.2), 244 (46.1), 231 (15.8), 215 (15.1), 164 (39.5), 135 (33.2), 91 (95.6), 77 (72.5). Anal. Calcd. for $\mathrm{C}_{27} \mathrm{H}_{23} \mathrm{~N}_{3} \mathrm{OS}$ (437.56): C, 74.11, H, 5.30, N, 9.60\%. Found: C, 74.18, H, 5.33, N, $9.69 \%$.

\section{2-(3,5-Diphenylcyclohex-2-enylidene)hydrazono)-4-meth} yl-3-phenyl-2,3-dihydrothiazole 8a

Brown powder, $\mathrm{mp} 98^{\circ} \mathrm{C}$, yield $82 \%$, IR $(\mathrm{KBr})$ (vmax, $\left.\mathrm{cm}^{-1}\right): 1609(\mathrm{C}=\mathrm{N}) .{ }^{1} \mathrm{H}-\mathrm{NMR} \quad \delta p p m=1.87\left(\mathrm{~s}, 3 \mathrm{H}, \mathrm{CH}_{3}\right)$, 2.33-2.43 (m, $\left.2 \mathrm{H}, \mathrm{C}_{4}-\mathrm{H}\right), 2.71-2.82\left(\mathrm{~m}, 2 \mathrm{H}, \mathrm{C}_{6}-\mathrm{H}\right), 2.91-2.96$ (m, $\left.1 \mathrm{H}, \mathrm{C}_{5}-\mathrm{H}\right), 3.02,3.11$ (d.d, $2 \mathrm{H}, \mathrm{CH}_{2}$ thiazole), 3.07-3.14 $(\mathrm{m}, 1 \mathrm{H}, \mathrm{CH}$ thiazole $), 6.76\left(\mathrm{~d}, 1 \mathrm{H}, J=3 \mathrm{~Hz}, \mathrm{C}_{2}-\mathrm{H}\right), 7.12-7.57$ (m, 15H, Ar-H). MS: (m/z, \%):436 (10.8), 435 (36.1), 304 (19.4), 266 (12.8), 189 (12.8), 176 (14.7), 141 (13.1), 135 (22.1), 118 (20.2), 104 (12.0), 93 (100), 77 (37.4). Anal. Calcd. For $\mathrm{C}_{28} \mathrm{H}_{25} \mathrm{~N}_{3} \mathrm{~S}$ (435.58): C, 77.21; H, 5.79; N, 9.65\%. Found: C, 77.18; H, 5.75; N, 9.63\%.

2-(3,5-diphenylcyclohex-2-enylidene)hydrazono)-3,4-dip henyl-2,3-dihydrothiazole $8 b$

Reddish brown powder, $\mathrm{mp} 104^{\circ} \mathrm{C}$, yield $85 \%$, IR $(\mathrm{KBr})$ $\left(\operatorname{vmax}, \mathrm{cm}^{-1}\right): 1608(\mathrm{C}=\mathrm{N}) .{ }^{1} \mathrm{H}-\mathrm{NMR} \delta \mathrm{ppm}=2.35-2.44(\mathrm{~m}$, $\left.2 \mathrm{H}, \mathrm{C}_{4}-\mathrm{H}\right), 2.73-2.82\left(\mathrm{~m}, 2 \mathrm{H}, \mathrm{C}_{6}-\mathrm{H}\right), 2.94-3.06(\mathrm{~m}, 1 \mathrm{H}$, $\left.\mathrm{C}_{5}-\mathrm{H}\right), 3.21,3.25$ (d.d, $2 \mathrm{H}, \mathrm{CH}_{2}$ thiazole), 4.06-4.12 (m, $1 \mathrm{H}$, $\mathrm{CH}$ thiazole), $6.62\left(\mathrm{~d}, 1 \mathrm{H}, J=3 \mathrm{~Hz}, \mathrm{C}_{2}-\mathrm{H}\right), 6.98-7.63(\mathrm{~m}, 20 \mathrm{H}$, Ar-H), MS: (m/z, \%): 497 (32.6), 486 (8.9), 456 (22.7), 437 (4.0), 406 (3.1), 381 (4.9), 304 (17.1), 262 (21.4), 247 (12.1), 143 (21.5), 118 (18.9), 93 (100), 77 (24.1). Anal. Calcd. For $\mathrm{C}_{33} \mathrm{H}_{27} \mathrm{~N}_{3} \mathrm{~S}$ (497.65): C, 79.64, H, 5.47, N, 8.44\%. Found: C, $79.75, \mathrm{H}, 5.38, \mathrm{~N}, 8.48 \%$.

\section{2-(3,5-Diphenylcyclohex-2-enylidene)hydrazono)-3-phen} ylthiazolidin-4-imine 9

Buff powder, mp $122^{\circ} \mathrm{C}$, yield $80 \%$, IR $(\mathrm{KBr})$ (vmax, $\left.\mathrm{cm}^{-1}\right): 3346(\mathrm{NH}), 1604(\mathrm{C}=\mathrm{N}) .{ }^{1} \mathrm{H}-\mathrm{NMR}$ oppm $=2.31-2.38$ $\left(\mathrm{m}, 2 \mathrm{H}, \mathrm{C}_{4}-\mathrm{H}\right), 2.65-73\left(\mathrm{~m}, 2 \mathrm{H}, \mathrm{C}_{6}-\mathrm{H}\right), 2.83-2.87(\mathrm{~m}, 1 \mathrm{H}$, $\left.\mathrm{C}_{5}-\mathrm{H}\right), 3.96\left(\mathrm{~s}, 2 \mathrm{H}, \mathrm{CH}_{2}\right.$ thiazole), $6.42(\mathrm{~d}, 1 \mathrm{H}, J=3 \mathrm{~Hz}$, $\left.\mathrm{C}_{2}-\mathrm{H}\right), 7.07-7.58(\mathrm{~m}, 15 \mathrm{H}, \mathrm{Ar}-\mathrm{H}), 8.03$ (s, $\left.1 \mathrm{H}, \mathrm{NH}\right)$. MS: (m/z, \%): 436 (10.8), 435 (36.1), 304 (19.4), 266 (12.8), 189 (12.8), 176 (14.7), 141 (13.1), 135 (22.1), 118 (20.2), 104 (12.0), 93 (100), 77 (37.4). Anal. Calcd. For $\mathrm{C}_{27} \mathrm{H}_{24} \mathrm{~N}_{4} \mathrm{~S}$ (436.57): C, 74.28, H, 5.54, N, 12.83\%. Found: C, 74.24, H, $5.48, \mathrm{~N}, 12.85 \%$.

1-(3,5-Diphenylcyclohex-2-enylidene)amino)-3-phenyl-2thioxodihydropyrimidine-4,6(1H,5H)-dione 10

To a stirred solution of $5(5.96 \mathrm{~g}, 0.015 \mathrm{~mol})$ in acetyl chloride $(10 \mathrm{~mL})$, malonic acid $(2.1 \mathrm{~g}, 0.02 \mathrm{~mol})$ was added. The reaction mixture was left for $2 \mathrm{~h}$ at ambient temperature and then heated for $4 \mathrm{hrs}$ at $50-55^{\circ} \mathrm{C}$. The contents were then poured onto crushed ice, cooled to $10^{\circ} \mathrm{C}$, and the separated solid was filtered off, dried and recrystallized from the ethanol to give the corresponding thiobarbituric acid derivatives 10 . Brown crystals, mp $136^{\circ} \mathrm{C}$, yield $74 \%$, IR $(\mathrm{KBr})$ $\left(\operatorname{vmax}, \mathrm{cm}^{-1}\right): 1685,1652(2 \mathrm{C}=\mathrm{O}), 1604(\mathrm{C}=\mathrm{N}) .{ }^{1} \mathrm{H}-\mathrm{NMR}$ $\delta$ ppm $=2.29-2.45\left(\mathrm{~m}, 2 \mathrm{H}, \mathrm{C}_{4}-\mathrm{H}\right), 2.76-2.89\left(\mathrm{~m}, 2 \mathrm{H}, \mathrm{C}_{6}-\mathrm{H}\right)$, 2.98-3.04 (m, 1H, $\left.\mathrm{C}_{5}-\mathrm{H}\right), 3.21\left(\mathrm{~s}, 2 \mathrm{H}, \mathrm{CH}_{2}\right), 6.17(\mathrm{~d}, 1 \mathrm{H}, J=$ $\left.3 \mathrm{~Hz}, \mathrm{C}_{2}-\mathrm{H}\right), 6.71-7.26$ (m, 15H, Ar-H). MS: (m/z, \%): 465 
(1.37), 458 (4.6), 437 (2.56), $420(2.42), 402(11.9), 377(5.1)$, $361(5.65), 347$ (4.32), $321(9.2), 302(17.7), 287(46.2), 262$ (21.0), 245 (100), 230 (99), 205 (24.7) 202 (24.0), 174 (9.9), 152 (12.7), 119 (34.1), 91 (52.5), 77 (56.6). Anal. Calcd. For $\mathrm{C}_{28} \mathrm{H}_{23} \mathrm{~N}_{3} \mathrm{O}_{2} \mathrm{~S}$ (465.57): C, 72.23, H, 4.98, N, 9.03\%. Found: C, 72.31, H, 5.06, N, 9.13\%.

Synthesis of arylidene derivatives 11a,b, 12a,b, and 13a,b

To a solution of 6,7 , or 10 (0.01 mole and aromatic aldehydes namely, benzaldehyde $(1.06 \mathrm{~g}, 0.01 \mathrm{~mol})$ or $p$-methoxybenzaldehyde $(1.36 \mathrm{~g}, 0.01 \mathrm{~mol})$ in ethanol $(20$ $\mathrm{mL}$ ), was added a few drops of piperidine and the reaction mixture was refluxed for $4 \mathrm{~h}$ then left to cool. The precipitate that formed was filtered off, washed with ethanol and recrystallized from ethanol to afford the corresponding derivatives $11 \mathrm{a}, \mathrm{b}, 12 \mathrm{a}, \mathrm{b}$ and $13 \mathrm{a}, \mathrm{b}$, respectively.

\section{4-Benzylidene-2-((-(3,5-diphenylcyclohex-2-enylidene)} hydrazono)-3-phenyl thiazolidin-5-one 11 a

Buff powder, mp $241^{\circ} \mathrm{C}$, yield $74 \%$, IR (KBr) (vmax, $\left.\mathrm{cm}^{-1}\right)$ : $1687(\mathrm{C}=\mathrm{O}), 1610(\mathrm{C}=\mathrm{N}) .{ }^{1} \mathrm{H}-\mathrm{NMR} \delta \mathrm{ppm}=2.31-$ 2.37(m, 2H, $\left.\mathrm{C}_{4}-\mathrm{H}\right), 2.65-73\left(\mathrm{~m}, 2 \mathrm{H}, \mathrm{C}_{6}-\mathrm{H}\right), 2.83-2.87(\mathrm{~m}, 1 \mathrm{H}$, $\left.\mathrm{C}_{5}-\mathrm{H}\right), 6.29\left(\mathrm{~d}, 1 \mathrm{H}, J=3 \mathrm{~Hz}, \mathrm{C}_{2}-\mathrm{H}\right), 6.81(\mathrm{~s}, 1 \mathrm{H}$, olefinic $\mathrm{CH})$, 6.99-7.54 (m, 20H, Ar-H). MS: (m/z, \%): 525 (7.2), 449 (19.1), 435 (9.2), 345 (22.4), 304 (11.6), 285 (32.5), 260 (40.9), 245 (93.4), 143 (43.6), 91 (100), 77 (56.5). Anal. Calcd. For $\mathrm{C}_{34} \mathrm{H}_{27} \mathrm{~N}_{3} \mathrm{OS}$ (525.66): C, 77.69, H, 5.18, N, 7.99\%. Found: C, 77.74, H, 5.25, N, 8.06\%.

2-((3,5-Diphenylcyclohex-2-enylidene)hydrazono)-4-(4methoxy benzylidene)-3-phenylthiazolidin-5-one 11b

White powder, mp $263^{\circ} \mathrm{C}$, yield $79 \%$, IR (KBr) (vmax, $\left.\mathrm{cm}^{-1}\right): 1684(\mathrm{C}=\mathrm{O}), 1603(\mathrm{C}=\mathrm{N}) .{ }^{1} \mathrm{H}-\mathrm{NMR} \delta \mathrm{ppm}=2.31$ 2.37(m, 2H, $\left.\mathrm{C}_{4}-\mathrm{H}\right), 2.65-73\left(\mathrm{~m}, 2 \mathrm{H}, \mathrm{C}_{6}-\mathrm{H}\right), 2.83-2.87(\mathrm{~m}, 1 \mathrm{H}$, $\left.\mathrm{C}_{5}-\mathrm{H}\right), 3.84\left(\mathrm{~s}, 3 \mathrm{H}, \mathrm{OCH}_{3}\right), 6.29\left(\mathrm{~d}, 1 \mathrm{H}, J=3 \mathrm{~Hz}, \mathrm{C}_{2}-\mathrm{H}\right), 6.81$ (s, 1H, olefinic CH), 6.99-7.54 (m, 19H, Ar-H). Anal. Calcd. For $\mathrm{C}_{35} \mathrm{H}_{29} \mathrm{~N}_{3} \mathrm{O}_{2} \mathrm{~S}$ (555.69): $\mathrm{C}, 75.65, \mathrm{H}, 5.26, \mathrm{~N}, 7.56 \%$. Found: C, 75.71, H, 5.34, N, 7.63\%.

(2Z,5Z)-5-Benzylidene-2-((E)-(3,5-diphenylcyclohex-2-e nylidene) hydrazono)-3-phenyl thiazolidin-4-one 12a

Pale yellow powder, mp $183^{\circ} \mathrm{C}$, yield $69 \%$, IR $(\mathrm{KBr})$ $\left(\operatorname{vmax}, \mathrm{cm}^{-1}\right)$ : $1687(\mathrm{C}=\mathrm{O}), 1605(\mathrm{C}=\mathrm{N}) .{ }^{1} \mathrm{H}-\mathrm{NMR} \delta \mathrm{ppm}=$ 2.31-2.37(m, 2H, $\left.\mathrm{C}_{4}-\mathrm{H}\right), 2.65-73\left(\mathrm{~m}, 2 \mathrm{H}, \mathrm{C}_{6}-\mathrm{H}\right), 2.83-2.87(\mathrm{~m}$, $\left.1 \mathrm{H}, \mathrm{C}_{5}-\mathrm{H}\right), 6.23\left(\mathrm{~d}, 1 \mathrm{H}, J=3 \mathrm{~Hz}, \mathrm{C}_{2}-\mathrm{H}\right), 7.03-7.54(\mathrm{~m}, 20 \mathrm{H}$, Ar-H), 7.78 (s, 1H, olefinic $\mathrm{CH})$. Anal. Calcd. For $\mathrm{C}_{34} \mathrm{H}_{27} \mathrm{~N}_{3} \mathrm{OS}$ (525.66): C, 77.69, H, 5.18, N, 7.99\%. Found: C, 77.76, H, 5.26, N, 8.03\%.

(2Z,5Z)-2-((E)-(3,5-Diphenylcyclohex-2-enylidene)hydra zono)-5-(4-methoxybenzylidene)-3-phenylthiazolidin-4-o ne $12 b$

Pale yellow powder, mp $206^{\circ} \mathrm{C}$, yield $71 \%$, IR $(\mathrm{KBr})$ $\left(\operatorname{vmax}, \mathrm{cm}^{-1}\right): 1681(\mathrm{C}=\mathrm{O}), 1604(\mathrm{C}=\mathrm{N}) . \mathrm{MS}:(\mathrm{m} / \mathrm{z}, \%): 555$ (4.3), 524 (12.7), 449 (6.5), 435 (26.8), 381 (12.4), 345 (28.6), 288 (32.7), 262 (56.1), 244 (39.1), 231 (25.4), 164 (41.3), 135 (28.5), 91 (100), 77 (76.4). Anal. Calcd. For $\mathrm{C}_{35} \mathrm{H}_{29} \mathrm{~N}_{3} \mathrm{O}_{2} \mathrm{~S}$ (555.69): C, 75.65, H, 5.26, N, 7.56\%. Found: C, 75.70, H, 5.28, N, 7.64\%.

5-Benzylidene-1-(3,5-diphenylcyclohex-2-enylideneamin
o)-3-phenyl-2-thioxodihydropyrimidine-4,6(1H,5H)-dion e 13a

Yellow crystal, mp $174^{\circ} \mathrm{C}$, yield $58 \%$, IR (KBr) (vmax, $\left.\mathrm{cm}^{-1}\right)$ : 1686,1653 $(2 \mathrm{C}=\mathrm{O}), 1602(\mathrm{C}=\mathrm{N}) . \mathrm{MS}:(\mathrm{m} / \mathrm{z}, \%): 553$ (12.7), 525 (3.9),476 (4.3),463 (6.2), 458 (4.6), 437 (2.56), 420 (2.42), 402(11.9), 377 (5.1), 361 (5.65), 347 (4.32), 321 (9.2), 302 (17.7), 287 (46.2), 262 (21.0), 245 (100), 230 (99), 215 (24.7) 202 (24.0), 174 (9.9), 152 (12.7), 119 (34.1), 91 (52.5), 77 (56.6). Anal. Calcd. For $\mathrm{C}_{35} \mathrm{H}_{27} \mathrm{~N}_{3} \mathrm{O}_{2} \mathrm{~S}$ (553.67): $\mathrm{C}$, 75.92, H, 4.92, N, 7.59\%. Found: C, 75.94, H, 4.93, N, $7.61 \%$.

1-(3,5-Diphenylcyclohex-2-enylideneamino)-5-(4-methox ybenzylidene)-3-phenyl-2-thioxodihydropyrimidine-4,6( 1H,5H)-dione 13b

Yellow powder, $\mathrm{mp} 216^{\circ} \mathrm{C}$, yield $84 \%$, IR (KBr) (vmax, $\left.\mathrm{cm}^{-1}\right): 1684,1651(2 \mathrm{C}=\mathrm{O}), 1602(\mathrm{C}=\mathrm{N}) .{ }^{1} \mathrm{H}-\mathrm{NMR} \delta \mathrm{ppm}=$ 2.32-2.39(m, 2H, $\left.\mathrm{C}_{4}-\mathrm{H}\right), 2.65-73\left(\mathrm{~m}, 2 \mathrm{H}, \mathrm{C}_{6}-\mathrm{H}\right), 2.83-2.87(\mathrm{~m}$, $\left.1 \mathrm{H}, \mathrm{C}_{5}-\mathrm{H}\right), 3.90\left(\mathrm{~s}, 3 \mathrm{H}, \mathrm{OCH}_{3}\right), 6.22\left(\mathrm{~d}, 1 \mathrm{H}, J=3 \mathrm{~Hz}, \mathrm{C}_{2}-\mathrm{H}\right)$, 7.02-7.56 (m, 19H, Ar-H), 8.21 (s, 1H, olefinic CH). Anal. Calcd. For $\mathrm{C}_{36} \mathrm{H}_{29} \mathrm{~N}_{3} \mathrm{O}_{3} \mathrm{~S}$ (583.70): C, 74.08, H, 5.01, N, 7.20\%. Found: C, 74.12, H, 5.03, N, 7.21\%.

\section{Synthesis of arylazo derivatives 14a,b, 15a,b and 16a,b}

A solution of sodium nitrite $(0.70 \mathrm{~g}$ in $10 \mathrm{~mL}$ water $)$ was gradually added to a well cooled $\left(0-5^{\circ} \mathrm{C}\right)$ solution of the aromatic amines namely; $p$-anisidine, $p$-chloroaniline $(0.01$ mmol) in concentrated $\mathrm{HCl}(3.0 \mathrm{~mL})$. The diazonium salt solution was added with continuous stirring to a cold $\left(0-5^{\circ} \mathrm{C}\right)$ solution of compounds 6,7 or $10(0.01 \mathrm{~mol})$ in pyridine $(30$ $\mathrm{mL})$. The reaction mixture was allowed to stir at $\left(0-5^{\circ} \mathrm{C}\right)$ for $2 \mathrm{hrs}$, and then the solid was collected by filtration. The crude products thus obtained, were dried and recrystallized from ethanol to give the corresponding arylazo derivatives $14 \mathrm{a}, \mathrm{b}, 15 \mathrm{a}, \mathrm{b}$ and $16 \mathrm{a}, \mathrm{b}$, respectively.

2-(3,5-Diphenylcyclohex-2-enylidene)hydrazono)-4-(4-m ethoxyphenyl) diazenyl)-3-phenylthiazolidin-5-one 14a

Yellow powder, mp $106^{\circ} \mathrm{C}$, yield $64 \%$, IR (KBr) (vmax, $\left.\mathrm{cm}^{-1}\right): 1683(\mathrm{C}=\mathrm{O}), 1605(\mathrm{C}=\mathrm{N}) .{ }^{1} \mathrm{H}-\mathrm{NMR} \quad \delta \mathrm{ppm}=$ 2.31-2.37(m, 2H, $\left.\mathrm{C}_{4}-\mathrm{H}\right), 2.65-73\left(\mathrm{~m}, 2 \mathrm{H}, \mathrm{C}_{6}-\mathrm{H}\right), 2.83-2.87(\mathrm{~m}$, $\left.1 \mathrm{H}, \mathrm{C}_{5}-\mathrm{H}\right), 3.51(\mathrm{~s}, 1 \mathrm{H}, \mathrm{CH}$ thiazolidine $), 3.82\left(\mathrm{~s}, 3 \mathrm{H}, \mathrm{OCH}_{3}\right)$, $6.64\left(\mathrm{~d}, 1 \mathrm{H}, J=3 \mathrm{~Hz}, \mathrm{C}_{2}-\mathrm{H}\right), 6.95-7.59$ (m, 19H, Ar-H). MS: (m/z, \%): 571 (4.1), 543 (0.4), 522 (0.7), 509 (0.8), 492 (1.49), 456 (2.29), 437 (37), 345 (9.5), 304 (34.5), 262 (31.3), 254 (50), 115 (45.1), 91 (100), 77 (48.4). Anal. Calcd. For $\mathrm{C}_{34} \mathrm{H}_{29} \mathrm{~N}_{5} \mathrm{O}_{2} \mathrm{~S}$ (571.69): C, 71.43, H, 5.11, N, 12.25\%. Found: C, 71.52, H, 5.18, N, 12.33\%.

\section{4-(4-Chlorophenyl)diazenyl)-2-(3,5-diphenylcyclohex-2-} enylidene) hydrazono)-3-phenylthiazolidin-5-one $14 \mathrm{~b}$

Red powder, $\mathrm{mp} 109^{\circ} \mathrm{C}$, yield $72 \%$, IR $(\mathrm{KBr})\left(\mathrm{vmax}, \mathrm{cm}^{-1}\right)$ : $1682(\mathrm{C}=\mathrm{O}), 1606(\mathrm{C}=\mathrm{N}) .{ }^{1} \mathrm{H}-\mathrm{NMR} \delta \mathrm{ppm}=2.31-2.37(\mathrm{~m}$, $\left.2 \mathrm{H}, \mathrm{C}_{4}-\mathrm{H}\right), 2.65-73\left(\mathrm{~m}, 2 \mathrm{H}, \mathrm{C}_{6}-\mathrm{H}\right), 2.83-2.87\left(\mathrm{~m}, 1 \mathrm{H}, \mathrm{C}_{5}-\mathrm{H}\right)$, $3.54(\mathrm{~s}, 1 \mathrm{H}, \mathrm{CH}$ thiazolidine $), 6.55\left(\mathrm{~d}, 1 \mathrm{H}, J=3 \mathrm{~Hz}, \mathrm{C}_{2}-\mathrm{H}\right)$, 6.99-7.68 (m, 19H, Ar-H). MS: (m/z, \%): 578 (2.18), 576 (3.61), 550 (2.85), 522 (1.1), 492 (18.3), 435 (9.2), 350 (13.2), 304 (15.2), 285 (14.1), 262 (40.9), 245 (93.4), 143 (43.6), 103 (26.3), 91 (100), 77 (56.5). Anal. Calcd. For 
$\mathrm{C}_{33} \mathrm{H}_{26} \mathrm{ClN}_{5} \mathrm{OS}$ (576.11): C, 68.80, H, 4.55, N, 12.16\%. Found: C, 68.83, H, 4.61, N, 12.26\%.

2-(3,5-Diphenylcyclohex-2-enylidene)hydrazono)-5-(4-m ethoxyphenyl) diazenyl)-3-phenylthiazolidin-4-one 15a

Red powder, $\mathrm{mp} 121^{\circ} \mathrm{C}$, yield $56 \%$, IR $(\mathrm{KBr})\left(\operatorname{vmax}, \mathrm{cm}^{-1}\right)$ : $1684(\mathrm{C}=\mathrm{O}), 1608(\mathrm{C}=\mathrm{N})$. MS: (m/z, \%): $571(8.4), 522$ (4.3), 509 (2.1), 492 (6.5), 435 (26.8), 381 (12.4), 345 (28.6), 288 (32.7), 262 (56.1), 244 (39.1), 231 (25.4), 164 (41.3), 135 (28.5), 91 (100), 77 (76.4). Anal. Calcd. For $\mathrm{C}_{34} \mathrm{H}_{29} \mathrm{~N}_{5} \mathrm{O}_{2} \mathrm{~S}$ (571.69): C, 71.43, H, 5.11, N, 12.25\%. Found: C, 71.37, H, $5.05, \mathrm{~N}, 12.28 \%$.

5-(4-Chlorophenyl)diazenyl)-2-(3,5-diphenylcyclohex-2enylidene) hydrazono)-3-phenylthiazolidin-4-one $15 \mathbf{b}$

Red powder, $\mathrm{mp} 121^{\circ} \mathrm{C}$, yield $60 \%, \mathrm{IR}(\mathrm{KBr})\left(\mathrm{vmax}, \mathrm{cm}^{-1}\right)$ : $1685(\mathrm{C}=\mathrm{O}), 1604(\mathrm{C}=\mathrm{N}) .{ }^{1} \mathrm{H}-\mathrm{NMR} \delta \mathrm{ppm}=2.31-2.37(\mathrm{~m}, 2 \mathrm{H}$, $\left.\mathrm{C}_{4}-\mathrm{H}\right), 2.65-73\left(\mathrm{~m}, 2 \mathrm{H}, \mathrm{C}_{6}-\mathrm{H}\right), 2.83-2.87\left(\mathrm{~m}, 1 \mathrm{H}, \mathrm{C}_{5}-\mathrm{H}\right), 3.51$ (s, 1H, CH thiazole), $6.52\left(\mathrm{~d}, 1 \mathrm{H}, J=3 \mathrm{~Hz}, \mathrm{C}_{2}-\mathrm{H}\right), 6.99-7.68$ (m, 19H, Ar-H). MS: (m/z, \%): 576 (61.3), 550 (37.1), 492 (43.6), 436 (100), 350 (22.3), 305 (12.7), 285 (21.4), 262 (39.6), 245 (76.9), 143 (54.5), 91 (91.3), 77 (43.1). Anal. Calcd. For $\mathrm{C}_{33} \mathrm{H}_{26} \mathrm{ClN}_{5} \mathrm{OS}$ (576.11): C, 68.80, H, 4.55, N, $12.16 \%$. Found: C, 68.83, H, 4.61, N, 12.26\%.

1 - (3 , 5 - Diphenylcyclohex - 2 - enylideneamino ) - 5 ( ( 4-methoxyphenyl)diazenyl)-3-phenyl-2-thioxodihydro pyrimidine -4 , 6-( $1 \mathrm{H}, 5 \mathrm{H})$-dione $16 \mathrm{a}$

Yellow powder, mp $121^{\circ} \mathrm{C}$, yield $63 \%$, IR (KBr) (vmax, $\left.\mathrm{cm}^{-1}\right): 1684,1653(2 \mathrm{C}=\mathrm{O}), 1604(\mathrm{C}=\mathrm{N}) .{ }^{1} \mathrm{H}-\mathrm{NMR} \delta \mathrm{ppm}=$ 2.29-2.45 (m, 2H, C $4-\mathrm{H}), 2.76-2.89$ (m, 2H, $\left.\mathrm{C}_{6}-\mathrm{H}\right), 2.98-3.04$ $\left(\mathrm{m}, 1 \mathrm{H}, \mathrm{C}_{5}-\mathrm{H}\right), 3.41(\mathrm{~s}, 1 \mathrm{H}, \mathrm{CH}), 3.82\left(\mathrm{~s}, 3 \mathrm{H}, \mathrm{OCH}_{3}\right), 6.17(\mathrm{~d}$, $\left.1 \mathrm{H}, J=4 \mathrm{~Hz}, \mathrm{C}_{2}-\mathrm{H}\right), 6.71-7.26(\mathrm{~m}, 19 \mathrm{H}, \mathrm{Ph}-\mathrm{H})$. Anal. Calcd. For $\mathrm{C}_{35} \mathrm{H}_{29} \mathrm{~N}_{5} \mathrm{O}_{3} \mathrm{~S}$ (599.70): C, 70.10, H, 4.87, N, 11.68\%. Found: C, 70.08, H, 4.85, N, 11.66\%.

5-(4-Chlorophenyl)diazenyl)-1-(3,5-diphenylcyclohex-2enylidene-amino)-3-phenyl-2-thioxodihydropyrimidine4,6-(1H,5H)-dione $16 \mathrm{~b}$

Yellow powder, mp $121^{\circ} \mathrm{C}$, yield $69 \%$, IR (KBr) (vmax, $\left.\mathrm{cm}^{-1}\right): 1682,1654(2 \mathrm{C}=\mathrm{O}), 1604(\mathrm{C}=\mathrm{N}) .{ }^{1} \mathrm{H}-\mathrm{NMR} \delta \mathrm{ppm}=$ 2.28-2.47 (m, 2H, C $4-\mathrm{H}), 2.79-2.91\left(\mathrm{~m}, 2 \mathrm{H}, \mathrm{C}_{6}-\mathrm{H}\right), 2.99-3.05$ $\left(\mathrm{m}, 1 \mathrm{H}, \mathrm{C}_{5}-\mathrm{H}\right), 3.44(\mathrm{~s}, 1 \mathrm{H}, \mathrm{CH}), 6.17\left(\mathrm{~d}, 1 \mathrm{H}, J=4 \mathrm{~Hz}, \mathrm{C}_{2}-\mathrm{H}\right)$, 6.86-7.54 (m, 19H, Ar-H). Anal. Calcd. For $\mathrm{C}_{34} \mathrm{H}_{26} \mathrm{ClN}_{5} \mathrm{O}_{2} \mathrm{~S}$ (604.12): C, 67.60, H, 4.34, N, 11.59 Found: C, 67.62, H, $4.36, \mathrm{~N}, 11.61 \%$.

\section{REFERENCES}

[1] S. P. Singh, S. S. Parmar, K. Raman, V. I. Stenberg, Chem. Rev. 81, 175 (1981).

[2] K. Mogilaiah, R. B. Rao, and G. R. Sudhakar, Indian J. Chem., 40B, 336 (2001).

[3] F. Aydogan, N. "Ocal, Z. Turgut, and C. Yolacan, Bull. Korean Chem. Soc., 22, 476 (2001).

[4] N. Cesur, Z. Cesur, N. Ergenc, M. Uzun, M., Kiraz, O. Kasimo glu, and D. Kaya, Arch. Pharm. (Weinheim), 327, 271 (1994).
[5] H. Liu, Z. Li, and T. Anthonsen, Molecules, 5, 1055 (2000).

[6] L. Bonnemaison, Dokl. Soobshch.-Mezhdunar. Kongr. Zashch. Rast., 3, 79 (1975), b) L. Bonnemaison, Chem. Abstr., $88,165425 x(1978)$.

[7] D. Silva and D. J. Themistocles, U.S. Patent 3,966,953 (1976), b) D. Silva and D. J. Themistocles, Chem. Abstr., 86, 29794y (1977).

[8] S. S. Parmar, C. Dwivedi, A. Chaudhari, and T. K. Gupta, J. Med. Chem., 15, 99 (1972).

[9] E. 'Ylhan and N. Ergenc, Arch. Pharm. (Weinheim), 325, 453 (1992).

[10] A. G"ursoy and N. Karali, IL Farmaco, 50b 857 (1995).

[11] A. Agarwal, S. Lata, K.K. Saxena, V.K. Srivastava, A. Kumar, Eur. J. Med. Chem. 41, 1223 (2006).

[12] V. G. Zubenko, L. Y. Ladnaya, N. M. Turkevich, and S. M. Tatchinkapustyak, Farm. Zh., 29, 78 (1974), b) V. G. Zubenko, L. Y. Ladnaya, N. M. Turkevich, and S. M. Tatchinkapustyak, Chem. Abstr. 82, 667r (1975).

[13] G. Danila and C. Radu, Rev. Med. Chir., 82, 127 (1978), b) G. Danila and C. Radu, Chem. Abstr., 90, 33767s (1979).

[14] A. F. Pavlenko and S. D. Moshchitskii, Khim. Geterotsikl, Soedin., 2, 259 (1967), b) A. F. Pavlenko and S. D. Moshchitskii, Chem. Abstr., 68, 114479x (1968).

[15] T. Takematsu, M. Furushima, Y. Hasegawa, M. Morioka, and T. Tsuchiyama, Japan Patent 72 43, 812 (1972), b) T. Takematsu, M. Furushima, Y. Hasegawa, M. Morioka, and T. Tsuchiyama, Chem. Abstr., 79,143522p (1973).

[16] A. Andolsek, B. Stanovnik, M. Tisler, M. Likar, and P. Schauer, J. Med. Chem., 14, 53 (1971).

[17] W. O. Foye and P. Tovivich, J. Pharm. Sci., 66, 1607 (1977).

[18] S. K. Mallick, A. R. Martin, and R. G. Lingard, J. Med. Chem., 14, 528 (1971).

[19] V. R. Solomon, W. Haq, K. Srivastava, K, S. K. Puri, S. B. Katti, J. Med. Chem., 50, 394 (2007).

[20] C. V. Kavitha, B. Basappa, S. N.Swamy, , K. Mantelingu, S. Doreswamy, M. A. Sridhar, J. S. Prasa, K. S. Rangappa Bioorg. Med. Chem., 14, 2290 (2006).

[21] T. J. Shah, Desai, V. A. Arkivoc, Xiv, 218 (2007).

[22] A. Kumar, S. Sharma, A. Archana, K. Bajaj, S. Sharma, H. Panwar, T. Singh, V. K. Srivastava, Bioorg. Med. Chem., 11, 5293 (2003).

[23] S. Sharma, T. Singh, R. Mittal, K. K. Saxena, V. K. Srivastava, A. Kumar, Arch. Pharm. Chem. Life Sci., 339, 145 (2006).

[24] V.P.M. Rahman, S. Mukhtar, W.H. Ansari, G. Lemiere, Eur. J. Med. Chem. 40, 173 (2005).

[25] K. Babaoglu, M.A. Page, V.C. Jones, M.R. McNeil, C. Dong, J.H. Naismith, R.E. Lee, Bioorg. Med. Chem. Lett. 13, 3227 (2003).

[26] V. Ravichandran, B.R. Prashantha Kumar, S. Sankar, R.K. Agrawal, Eur. J. Med. Chem. 44, 1180 (2009). 
[27] H. A. Ead, S. O. Abdallah, N. A. Kassab, N. H. Metwalli, and Y. E. Saleh, Arch. Pharm. (Weinheim), 15, 99 (1972).

[28] M. A. Metwally, E. Abdel-Latif and F. A. Amer Sulfur Lett. 26119 (2003).

[29] M. A. Metwally, E. M. Keshk, A. Fekry and H. A. Etman Phosphorus, Sulfur and Silicon 179, 1 (2004).

[30] M. A. Metwally, E. M. Keshk, A. Fekry and H. A. Etman J. Chem. Res. 602 (2004).

[31] S. I. El-Desoky, H. A. Etman, S.B. Bondok, A. A. Fadda and M. A. Metwally, Sulfur Letters, 25, 199 (2002).

[32] S. I. El-Desoky. S. B. Bondock, H. A. Etman A. A. Fadda and M. A. Metwally, Sulfur Letters, 26, 127 (2003).

[33] M. A. Metwally, A. Metwally, E. Abdel-Galil and F. A. Amer,
Monatshefte fur Chemie, 139, 35, (2008).

[34] M. A. Metwally, E. Abdel-Galil, A. Metwally, and F. A. Amer Dyes Pigments 92, 902, (2012).

[35] A. sammour, M. T. Elzimaity and A. Abdel-Maksoud J. Chem U. A. R. 12, NO 4. 481-492 (1969).

[36] Y. Ohishi, T. Mukai, M. Nagohora, M. Yajima, N. Kajima, K. Miyohara, T. Tikano, Chem. Pharm. Bull. 38, 1911 (1990).

[37] Y. Mamosa, K. Megura, H.Ikeda, C. Hatamaka, S. Oi. T. Sohda, Chem. Pharm. Bull. 39, 1440 (1991).

[38] M. A. Metwally, E. Abdel-Latif, F. A. Amer, G. Kaupp Dyes Pigments 60, 249, (2004).

[39] M. A. Metwally, E. Abdel-Latif, A. M. Khalil, F. A. Amer, G. Kaupp Dyes Pigments 62, 181, (2004). 